\title{
UNCERTAINTY AND SENSITIVITY ANALYSIS IN TURBULENT PIPE FLOW SIMULATION
}

\author{
ZHIHAO DING ${ }^{1}$, KARINE TRUFFIN ${ }^{1}$, STEPHANE JAY ${ }^{1}$ AND DELPHINE \\ SINOQUET ${ }^{1}$ \\ ${ }^{1}$ IFP Energies Nouvelles \\ 1-4 avenue de Bois-Préau, Rueil-Malmaison 92852, France \\ E-mail : \{zhihao.ding, karine.truffin, stephane.jay, delphine.sinoquet\}@ifpen.fr
}

Key words: Uncertainty Quantification, Large-Eddy Simulation, Wall modeling, Surrogate modeling

\begin{abstract}
In this study, we would like to evaluate and improve the performance of Wall-Modeled LargeEddy Simulation (WMLES) on the modeling of a pipe flow for which Direct Numerical Simulation (DNS) data is available [1] and considered as a reference for further comparisons.

Models used in WMLES may raise problems of accuracy which come from the uncertain values of model parameters and model simplifications. In this study, we focus firstly on the impact of the model parameter uncertainties on the simulation results, and then on the reduction of these uncertainties via data calibration. These studies using sampling-based approaches can be unaffordable when coupled with a high-fidelity simulation that requires several CPU hours for a single execution. To reduce the computational cost while maintaining a target accuracy, we propose to build surrogate models based on Gaussian Processes for simulations outputs, and replace the simulator for evaluating the large size sampled sets.
\end{abstract}

For this study, a CFD-UQ methodology is developed which couples our internal UQ tool and a CFD solver. It has been applied on a turbulent pipe flow case that allows us to validate its implementation.

\section{INTRODUCTION}

In the last decades, supported by the rapid development of computational capacity and the improvement of mathematical modeling, a significant increased usage of numerical simulation tools like Computational Fluid Dynamics (CFD) for the analysis and design of numerous engineering applications can be observed. The use of CFD in automobile engineering becomes more common, especially the Large-Eddy Simulation (LES), for its ability to reproduce the numerous coupled phenomena in internal combustion engines. However, in such simulations, uncertainties can arise from different inputs of the simulation, including material properties, initial and boundary conditions, physical models and numerical resolution, which question the quality of the results obtained and disturb the associated analysis.

To obtain reliable results, it is necessary to study the impact of all these uncertainties onto the final solution. A possible approach is the application of Uncertainty Quantification (UQ) tools, which allows to propagate uncertainties related to different factors (inputs and parameters, ...) of a simulator (i.e. the computational code) to its outputs. The use of UQ in CFD application has attracted attention from various domains, typically in nuclear engineering where CFD is more and more used as a tool for design and safety issues and related uncertainties need to be well controlled [2], and also in aerospace applications [3] and aircraft design [4]. UQ has been introduced in experimental research [5] in automobile engineering, while numerical work with UQ is still challenging on real systems like internal combustion 
engines. Therefore our motivation is to develop a feasible UQ methodology for engine CFD applications.

UQ methods can be generally divided into 2 categories : intrusive and non-intrusive UQ methods. In intrusive approaches, the stochastic description of the parameter is integrated within the numerical formulation which may exhibits algorithmic difficulties. In contrast, non-intrusive approaches are sampling based, and the simulator is used as a black box. Therefore they are usually used in CFD applications.

The most known non-intrusive UQ method is the Monte Carlo sampling which is very straightforward and easy to implement. However, it requires a large number of simulator runs to achieve accurate statistical results due to its low convergence rate, and becomes intractable for CFD applications. One technique is to replace the CFD code by surrogate models characterized by fast response time. Only a limited number of simulations is necessary to construct surrogate models which provide an approximation of the relationship between simulation input and output vectors. Further UQ analysis can fully rely on predictions provided by these surrogate models. A popular surrogate model in UQ is based on Gaussian Processes (GP) which allow flexible approximations with an intrinsic control of estimation errors [6].

The present study aims to develop a non-intrusive CFD-UQ methodology based on existing UQ techniques to quantify accuracy, robustness and sensitivity of CFD simulations. Analysis is based on surrogate models of Quantities of Interest (QoIs) extracted from simulations and chosen for UQ study. Its application to engine simulations remains difficult as the engine flow is strongly non-stationary and complex, and uncertainties can come from not only physical models used in simulation for which parameters are not always known and can be case-dependent, but also from boundary conditions obtained from experimental measurements for which uncertainties are not well estimated. For validation purposes, this methodology is applied on a relatively simple case : a turbulent pipe flow, which features typical conditions of engine flows

The structure of this pa and in Section 3 detailed is provided. Section 4 pres
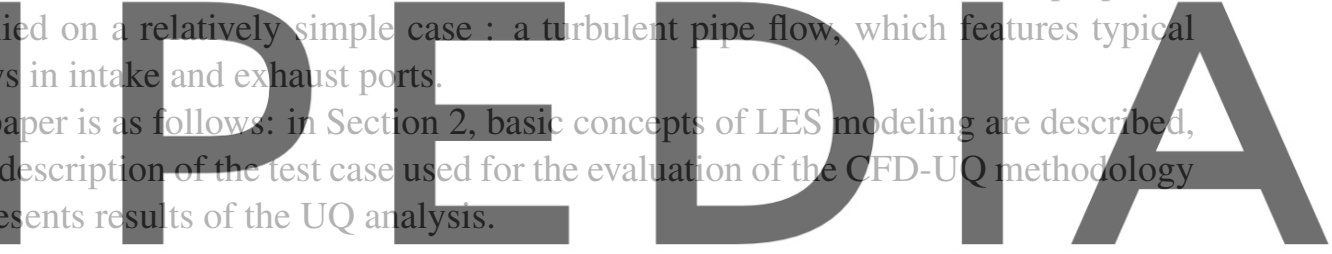

Register for free at https//www.scipedia.com to download the version without the watermark In LES, filtered Navier-Stokes equations are solved. The Favre-filtered momentum equation for compressible flows can be written as follows [7]:

$$
\frac{\partial \bar{\rho} \tilde{u}_{i}}{\partial t}+\frac{\partial \bar{\rho} \tilde{u}_{i} \tilde{u}_{j}}{\partial x_{j}}=-\frac{\partial \bar{p}}{\partial x_{i}}+\frac{\partial \bar{\tau}_{i j}}{\partial x_{j}}-\frac{\partial}{\partial x_{j}}\left(\bar{\rho}\left(\widetilde{u_{i} u_{j}}-\tilde{u}_{i} \tilde{u}_{j}\right)\right)
$$

where $\bar{\rho}$ is the density, $\bar{p}$ is the pressure, $u_{i}$ the $i^{\text {th }}$ component of the velocity field, $\bar{\tau}_{i j}$ is the viscous shear stress tensor and $\bar{\rho}\left(\widetilde{u_{i} u_{j}}-\tilde{u_{i}} \tilde{u_{j}}\right)$ is the unresolved subgrid stress tensor $\tau_{i j}^{S G S} \cdot \tau_{i j}^{S G S}$ needs special modeling to close the equation, therefore subgrid stress model is necessary in LES.

\subsection{Closure of the Favre-filtered momentum equation}

The Smagorinsky SGS model is chosen for SGS modeling in this study. It is the simplest model and has been proven to perform reasonably well [8]. The basic principle of such a model is to account for the additional diffusivity inherited from the turbulent flow structures that are smaller than the filter size, by adding explicitly in the conservation equations an additional, non-physical viscosity, called turbulent or eddy viscosity $v_{t}$. It assumes that it is possible to express the subgrid stress tensor $\tau_{i j}^{S G S}$ in a similar way 
to the shear stress tensor $\bar{\tau}_{i j}$ following :

$$
\begin{aligned}
\bar{\tau}_{i j} & =2 \bar{\rho} \vee\left(\tilde{S}_{i j}-\frac{1}{3} \delta_{i j} \tilde{S}_{k k}\right) \\
\tau_{i j}^{S G S}-\frac{1}{3} \delta_{i j} \tau_{k k}^{S G S} & =2 \bar{\rho} v_{t}\left(\tilde{S}_{i j}-\frac{1}{3} \delta_{i j} \tilde{S}_{k k}\right)
\end{aligned}
$$

where $\tilde{S}_{i j}$ is the strain rate tensor computed as $\tilde{S}_{i j}=\frac{1}{2}\left(\frac{\partial \tilde{u}_{i}}{\partial x_{j}}+\frac{\partial \tilde{u}_{j}}{\partial x_{i}}\right)$.

Using the Boussinesq hypothesis [8], the eddy viscosity $v_{t}$ is modeled as $v_{t}=\left(C_{s} \Delta\right)^{2} \sqrt{2 \tilde{S}_{i j} \tilde{S}_{i j}}$, where $\Delta$ is the filter size of LES and $C_{s}$ the Smagorinsky constant. The value of $C_{s}$ is not universal and depends on the flow's nature. A typical value of $C_{s}$ is 0.18 which is determined from the homogeneous isotropic turbulence. In practice, its value may vary from 0.1 to 0.23 [9].

\subsection{Wall shear stress modeling}

When applying a wall-function boundary condition, the wall-normal velocity is set to zero and the wall shear stress is imposed. The friction velocity $u_{\tau}$ is obtained by solving iteratively the logarithmic law of the wall :

$$
u^{+}=\frac{1}{\kappa} \ln y^{+}+B, y^{+}>30
$$

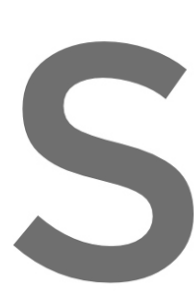

where $u^{+}=\tilde{U} / u_{\tau}$ and $y$

the kinematic viscosity

$\tau_{w}$ will then be used to

The empirical constants

constant, $B$, are generally
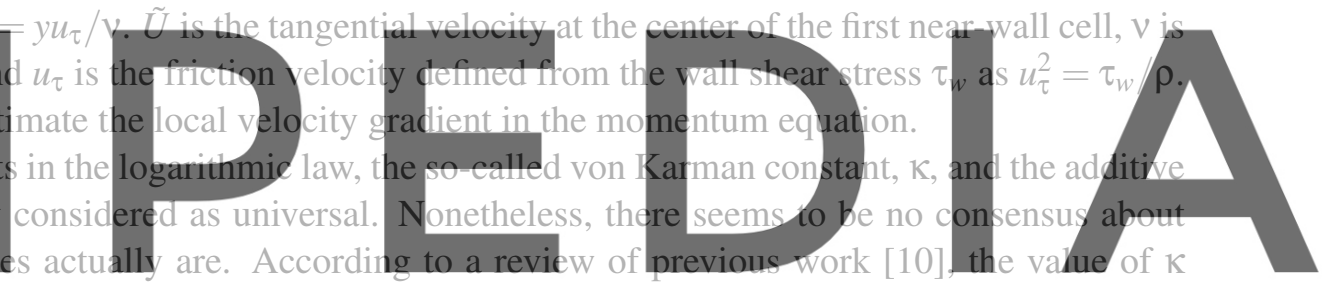

may vary between 0.3 and 0.45 , and that of $B$ may range from 4 to 6. Most values are extracted from

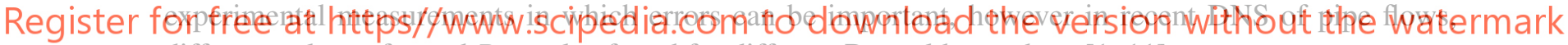
different values of $\mathrm{K}$ and $B$ are also found for different Reynolds numbers [1,11].

\section{Turbulent pipe flow}

\subsection{Presentation of the test-case}

The test-case chosen for the UQ study is a fully developed turbulent pipe flow at Reynolds number $\mathrm{Re}=44000$. Direct Numerical Simulation results on the same case obtained by Wu and Moin [1] can be used as reference data for the UQ study. This canonical case has been extensively used in the literature to study the physics of wall-bounded turbulence and also to develop and test numerical algorithms due its simple nature. Additionally, same kind of flows exist also in internal combustion engine, in both intake and exhaust ports (typical $R e$ is in the order of $10^{4}$ [12]). UQ study on this case helps us to prepare the UQ application on engine cases in the future.

The pipe's radius is $R=D / 2=5 \mathrm{~mm}$ and its length is $15 R=75 \mathrm{~mm}$ as recommended in [1]. An air flow at $300 \mathrm{~K}$ and $1 \mathrm{~atm}$ is driven by fixed mass flux and is periodic in axial direction $z$, the corresponding averaged bulk velocity $U_{b}$ is $69.3 \mathrm{~m} / \mathrm{s}$. The pipe wall is set to be isothermal and its temperature is fixed to $300 \mathrm{~K}$. The pipe flow simulations are carried out using commercial CFD solver CONVERGE V2.4 
[13]. For this high Reynolds number flow, WMLES is performed as it gives a good compromise between computational cost and accuracy. Detailed simulation set-up is described in the following sections.

\subsection{Simulation set-up}

The simulation is performed using a $2^{\text {nd }}$ order Crank-Nicolson scheme for temporal integration and a $2^{\text {nd }}$ order central scheme for spatial discretization. A CFL number based on convective velocity of 0.3 is used to limit the time step. The Cartesian mesh used is presented in Figure 1, as we can notice, cells close to the pipe wall are set 2 times larger than those in the inner region so that center of wall cells is located in the logarithmic region of the boundary layer. Finer grid size is used in outer regions where gradients of the filtered velocity need to be resolved. Turbulent fluctuations are located in the inner region and vanish in the near-wall region as it can be observed in Figure 1.
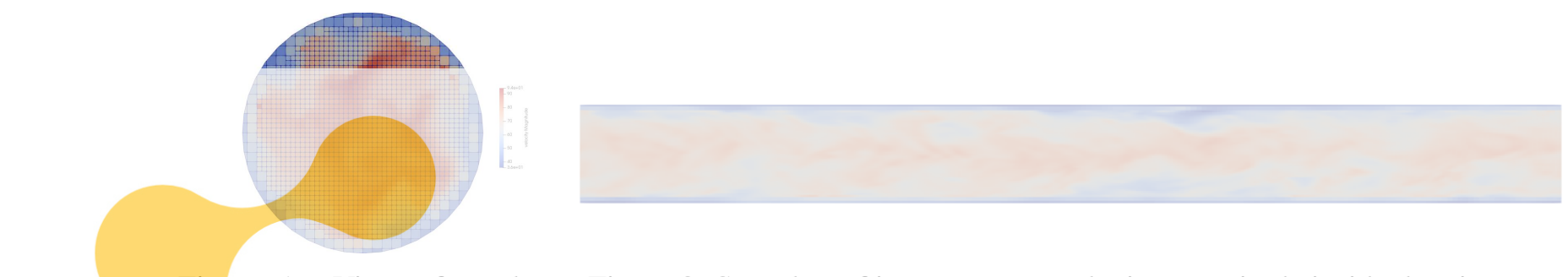

Figure 1: View of mesh on Figure 2: Snapshot of instantaneous velocity magnitude inside the pipe transversal section of the pipe
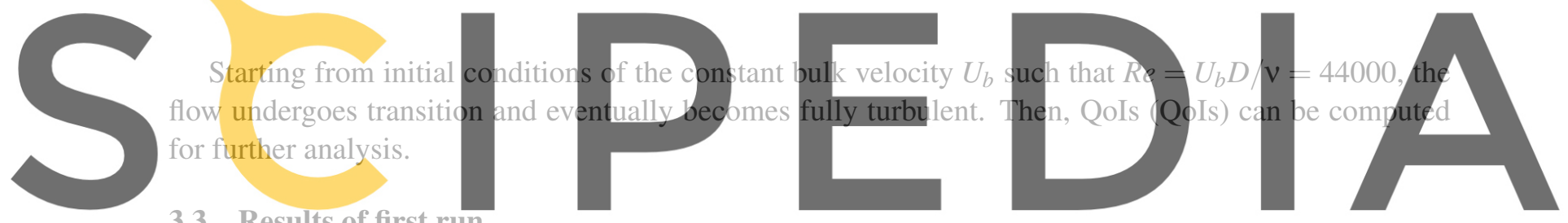

3.3 Results of first run

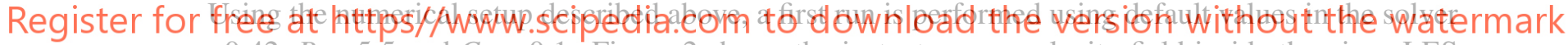
$\mathrm{K}=0.42, B=5.5$ and $C_{s}=0.1$. Figure 2 shows the instantaneous velocity field inside the pipe. LES well captures detailed turbulent structures of different scales.

Instantaneous velocity does not permit to characterize the flow, therefore the averaged axial velocity $\tilde{U}(r)$ in the radial direction $r$ is computed as the averaged flow is axially symmetric. Once the flow becomes fully turbulent, the simulation continues for an additional time of $300 R / U_{b}$, allowing a particle to travel 20 times along the pipe at the bulk velocity $U_{b}$. Statistics are collected for each time step during this period. Finally, velocity is averaged over time and axial direction of the flow for a better convergence. In the following, $\tilde{U}(r)$ states for its the time averaged value.

The averaged axial velocity $\tilde{U}$ as a function of the normalized wall distance is presented in Figure 3, and the corresponding dimensionless profile is presented in Figure 4. The center of the first cell is located at $y^{+}=36$, well inside the log-law region. With the current configuration, the velocity profile is already comparable with that of DNS. Considering DNS data as reference, the relative error of our simulation on $\tilde{U}$ can be quantified by a relative error $\varepsilon_{\tilde{U}}$ defined as $\varepsilon_{\tilde{U}}=\left|\left(\tilde{U}-\tilde{U}_{r e f}\right) / \tilde{U}_{\text {ref }}\right|$. The global error on the profile we have in the first run is of $2.1 \%$. 


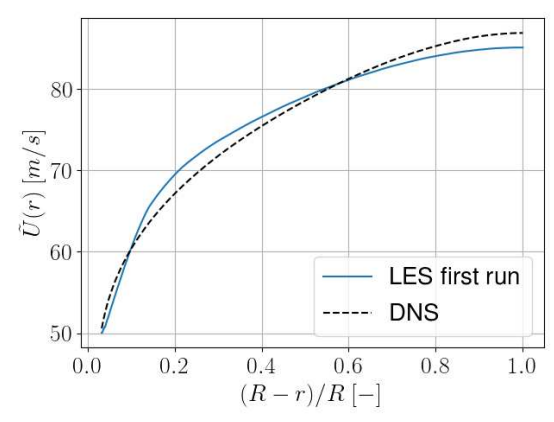

Figure 3: Mean profile $\tilde{U}$

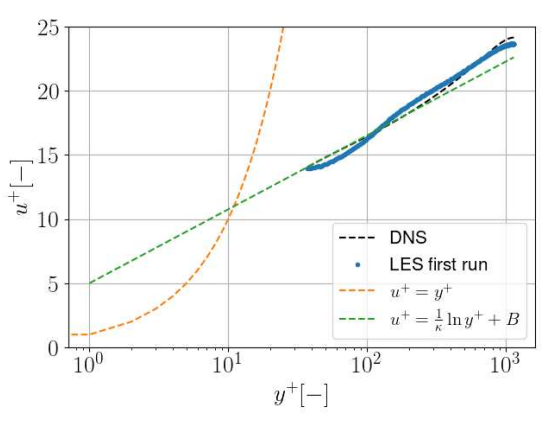

Figure 4: Dimensionless mean profile $\tilde{U}^{+}$

The momentum equation of the mean flow can be written as :

$$
\frac{d \bar{\tau}}{d r}=-\frac{d \bar{p}}{d z}
$$

In the periodic fully turbulent flow, the mean pressure gradient is uniform across the flow, thus the mean shear stress is also constant. Knowing values of $\bar{\tau}$ at the pipe center $\bar{\tau}(0)=0$ and at the wall $\bar{\tau}(R)=\tau_{w}$, the mean shear stress $\bar{\tau}$ is obtained as $\bar{\tau}(r)=\tau_{w} \cdot r / R$.

The total shear stress $\tilde{\tau}_{\text {tot }}$ is the sum of 3 contributions :
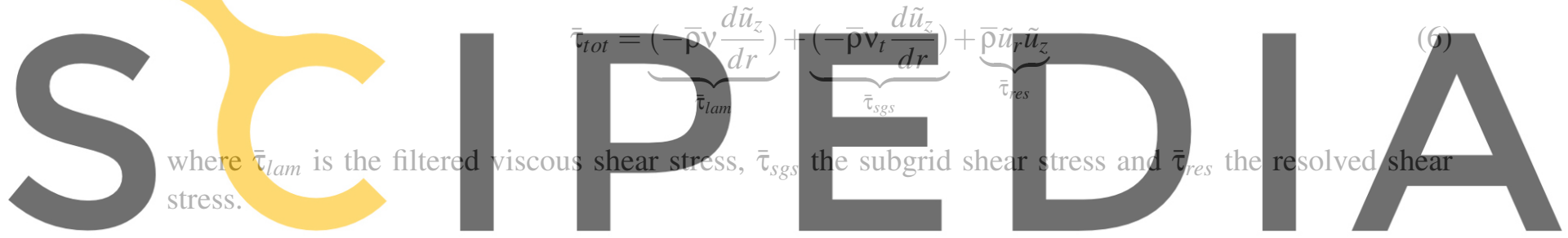

Register for free at https//www.scipedia.com to download the version without the watermark

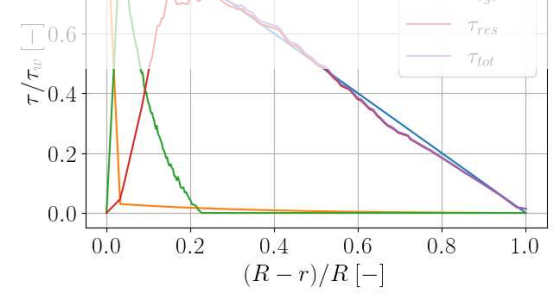

Figure 5: Shear stress distribution in wall normal direction

The time-averaged shear stress budget is presented in Figure 5, the analytical expression of the total shear stress is also plotted for comparison. Far from the wall, the total shear stress, mainly contributed by the turbulent shear stress, follows well the analytical expression. The wall shear stress is completely viscous, while in the inner region of the pipe the viscous stress is negligible. In the near wall region, the shear stress is mainly due to the subgrid stress model, which also becomes negligible far off the wall. In the vicinity of the wall, the total shear stress deviates from the analytical expression, which may be 
related to the jump of cell size in this area which disturbs the estimation of the local gradient and the resolution of the momentum equation.

\section{UNCERTAINTY AND SENSITIVITY ANALYSIS ON PIPE FLOW SIMULATION}

In this section, the CFD-UQ methodology is evaluated on the test case described in the previous section. Only uncertainties from the 2 physical models used in LES are studied.

\subsection{Methodology}

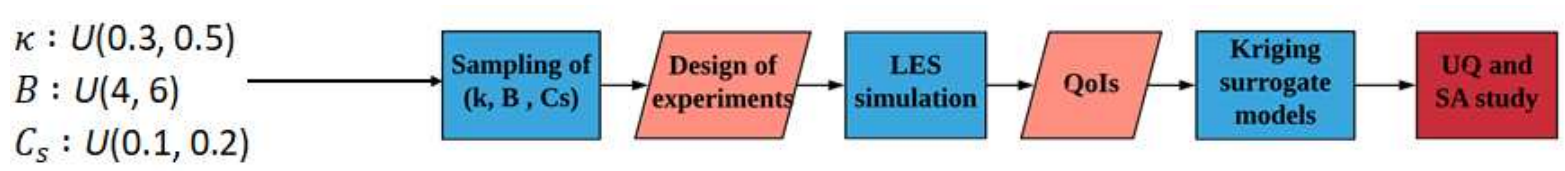

Figure 6: Workflow of CFD-UQ methodology

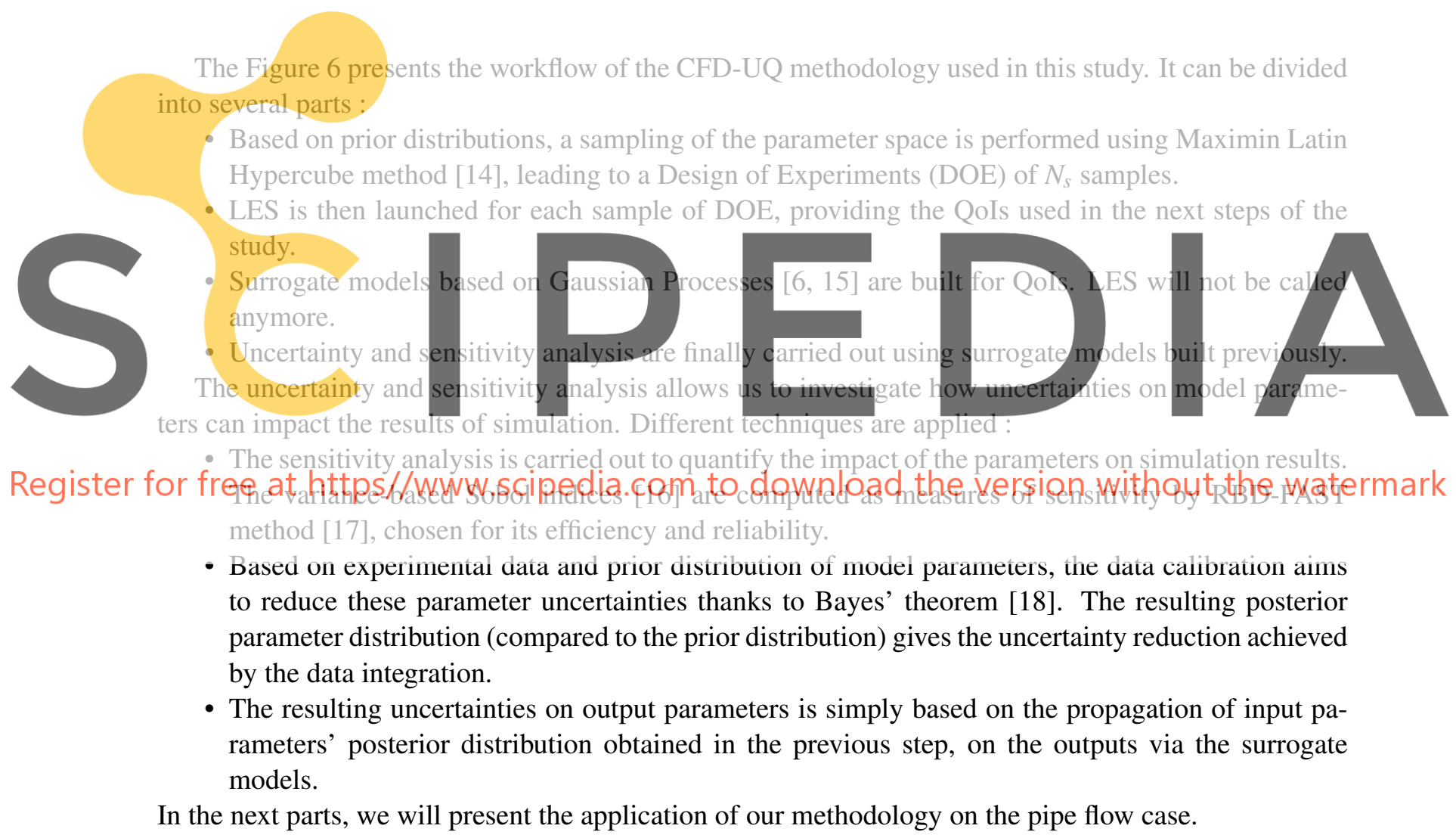

\subsection{Design of experiments}

We focus on the uncertainties introduced by the constant parameters of the SGS tensor and of the lawof-the-wall used in LES of pipe flow. Consistently with ranges of values found in the literature $[9,10]$, we define for each parameter a uniform prior distribution as follows : $\kappa \in U(0.3,0.5), \quad B \in U(4,6), \quad C_{s} \in$ $U(0.1,0.2)$. 
Based on prior distributions, a DOE of 400 samples is generated. Similar number of samples is used for another study with also 3 parameters and associated surrogate models showed good quality [19].

\subsection{Surrogate modeling}

LES is then performed for all samples to compute QoIs defined in Section 3.3 : averaged velocity profile $\tilde{U}(r)$ and the averaged error $\varepsilon_{\tilde{U}}$. The results are presented in Figure 7 and Figure 8. It can be seen that $\tilde{U}(r)$ varies significantly with respect to the 3 parameters and the corresponding error as well. The reference curve of $\tilde{U}(r)$ lies well inside the variation range. The maximal $\varepsilon_{\tilde{U}}$ can reach more than 0.15 which represents an important deviation compared to the reference data.

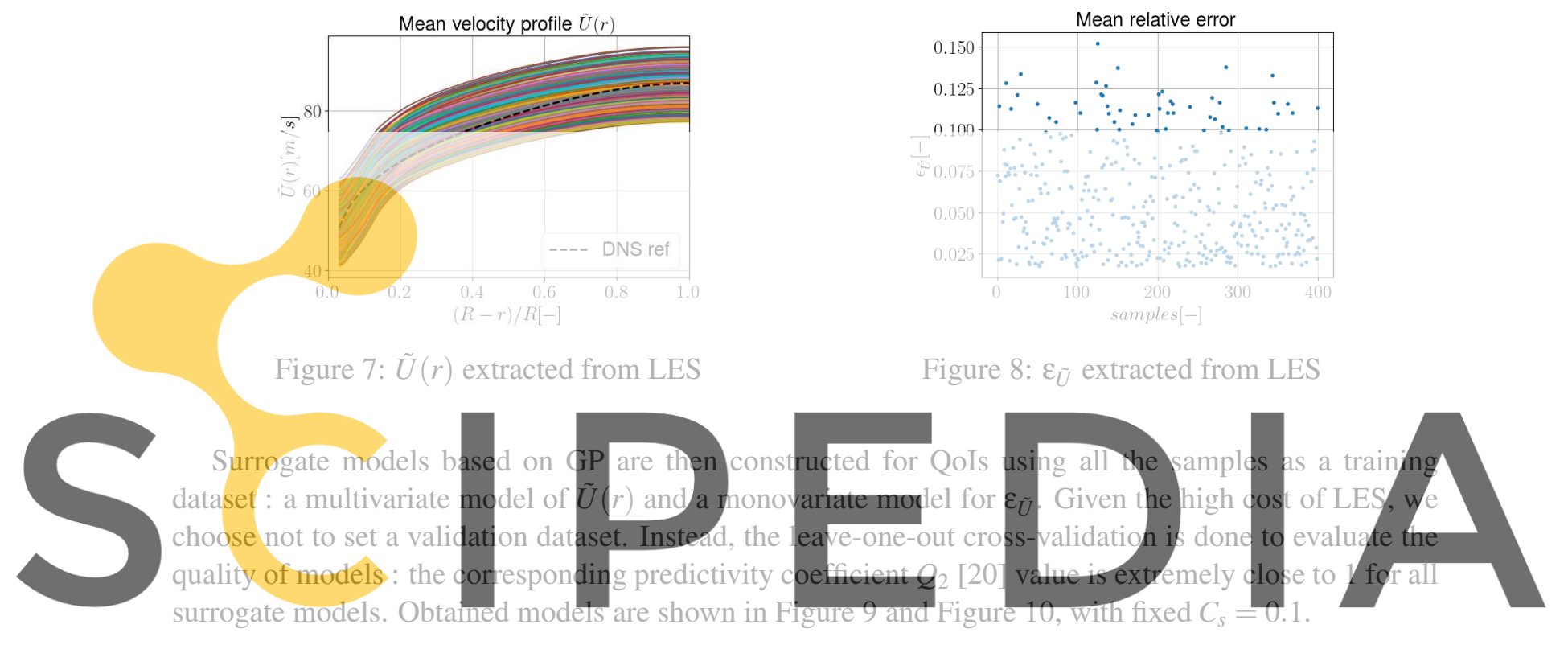

Register for free at https//www.scipedia.com to download the version without the watermark

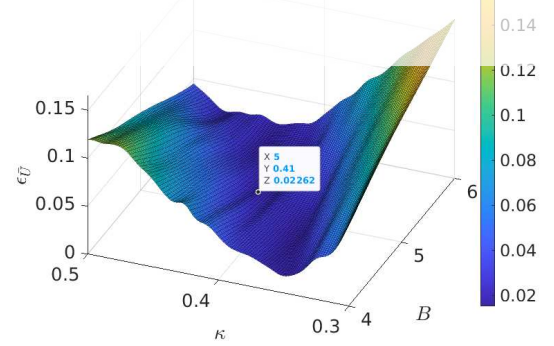

Figure 9: Response surface of $\varepsilon_{\tilde{U}}$

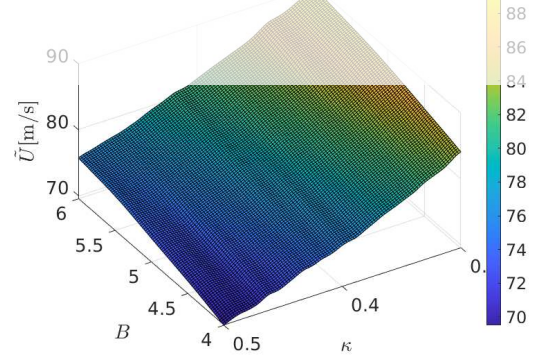

Figure 10: Response surface of $\tilde{U}(r)$ for $r=R / 2$

Figure 9 shows us that the response surface of $\varepsilon_{\tilde{U}}$ is of valley shape. $\varepsilon_{\tilde{U}}$ is minimized for $\kappa$ and $B$ located at the bottom of the valley. Additionally, a couple of $\kappa=0.41$ and $B=5$ is found in this area and corresponds to values found from pipe flow experiments [21], which confirms our simulated results. The response surface of $\tilde{U}(r)$ at $r=R / 2$ shown in Figure 10 is almost flat, and the impact of $\kappa$ and $B$ on the velocity at this point is obvious : smaller value of $B$ and larger value of $\kappa$ lead to lower velocity and 
$\kappa$ seems to have a relatively more important role.

\subsection{Sensitivity analysis}

The purpose of sensitivity analysis is to rank the input parameters according to their impact on the simulated outputs. In this part, variance-based Sobol indices are computed, which allows the quantification of the impact of the 3 parameters on the QoIs extracted from LES.

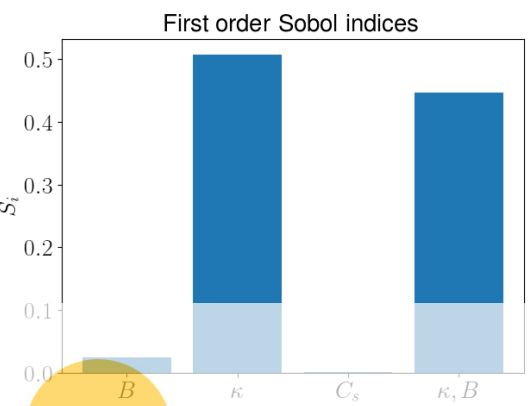

Figure 11: Sobol indices of $\varepsilon_{\tilde{U}}$

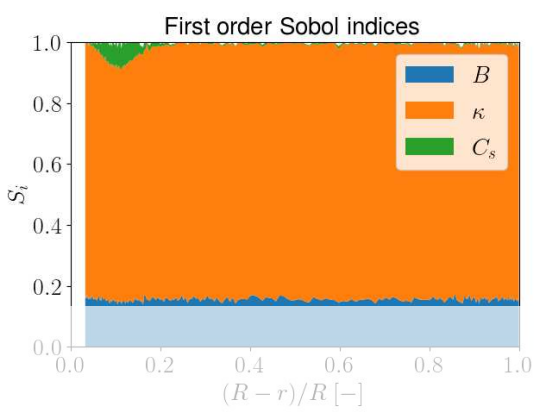

Figure 12: Sobol indices of $\tilde{U}(r)$

The sensitivity analysis is firstly done for $\varepsilon_{\tilde{U}}$. Sobol indices are plotted in Figure 11 . It is clear that $\kappa$ is the most influential parameter for $\varepsilon_{\tilde{U}}$. B alone doesn't have a significant influence but combined with $\mathbb{K}$ it also takes an important part in the variations of $\varepsilon_{\tilde{U}}$. Relatively the Smagorinsky constant $C_{S}$ has litte impact on the variations of $\varepsilon_{\tilde{U}}$.

The same study is then carried out for the QoI

velocity along the profile on the velocity profile a distance. We observe that $k$ is stitt the most criticat
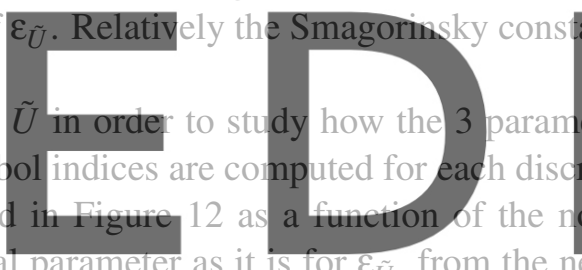
to the pipe center. The influence of $B$ is relatively less significant and is constant along the profile. $C$,

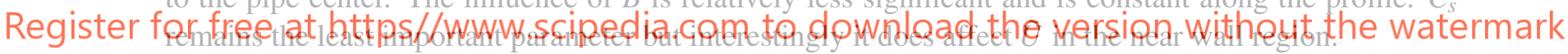

As a reminder, the contribution from the subgrid scale in total shear stress $\bar{\tau}_{s g s}$ is dominant in the near wall region, as we can see in Figure 5. Moreover, the region in which $C_{S}$ impacts the most coincides with the one in which $\bar{\tau}_{s g s}$ is prevalent among all the contributions. This can be explained by the fact that $\bar{\tau}_{s g s}$ depends on the value of $C_{S}$ as it is proportional to the eddy viscosity modeled by the SGS model. In fact, the turbulent viscosity given by the Smagorinsky model increases when approaching the wall to reach a value similar to the one obtained by the Prandtl mixing-length model [22]. Therefore, $C_{s}$ can directly affect the shear stress of flow and can finally affect the velocity profile, though its impact is relatively weak compared with parameters of the law-of-the-wall model.

We can also compute the momentum thickness $\theta$ of the boundary layer using the following formula :

$$
\theta=\int_{0}^{R} \frac{\tilde{U}(y)}{\tilde{U}_{c}}\left(1-\frac{\tilde{U}(y)}{\tilde{U}_{c}}\right) d y, y=R-r
$$

where $\tilde{U}_{c}$ is the velocity at the pipe center. Using the reference $\tilde{U}$ from DNS, the thickness $\theta$ equals to $0.089 R$, i.e $\theta^{+}=106$ in dimensionless scale, which is larger than the dimensionless wall distance of the 
first wall cells $\left(y^{+}=35\right.$ in average). The maximal Sobol indice of $C_{s}$ locates at $0.1 R$ which is of the same order of magnitude as $\theta$.

\subsection{Data calibration}
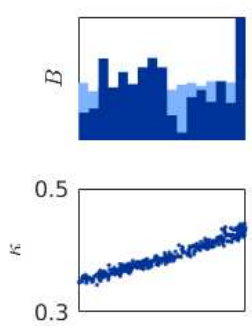

0.2

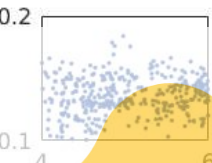

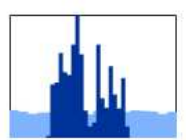

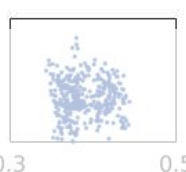

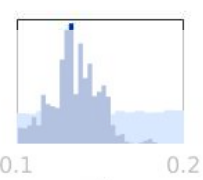

0.2

Figure 13: Prior and posterior distributions of inputs

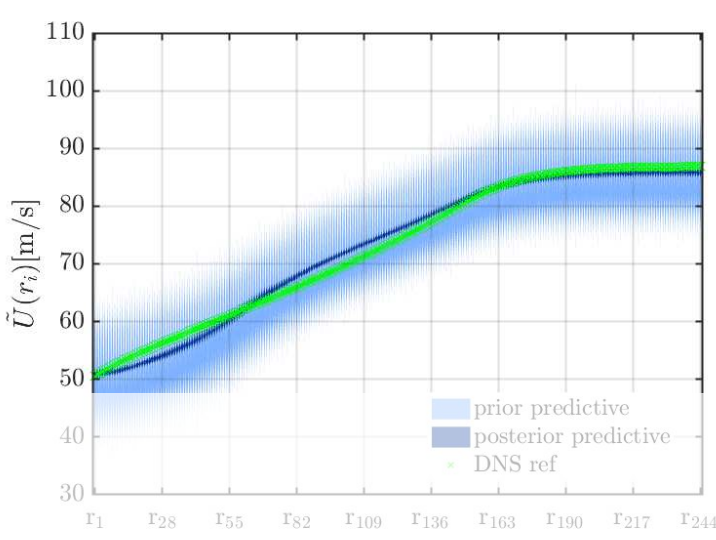

Figure 14: Uncertainty propagation on $\tilde{U}(r)$

As we can see in Fi

important. To reduce thest

reference data. The result

and posterior after calibra

joint distributions between

for the 3 parameters as
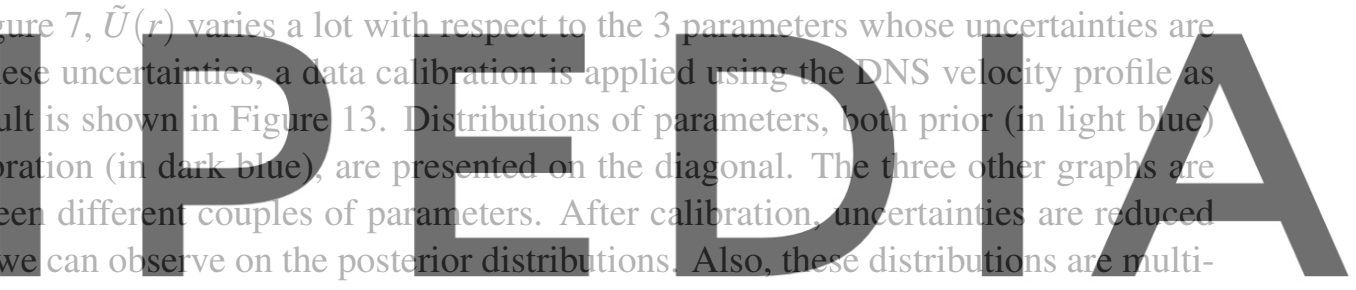

modal meaning that several values of the parameters are the most likely to lead to a good data calibration

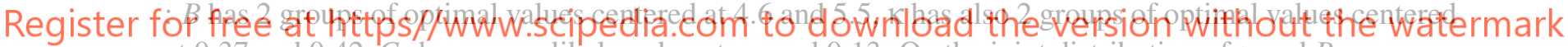
at 0.37 and $0.42, C s$ has a very likely value at around 0.13 . On the joint distribution of $\kappa$ and $B$, a narrow

zone appears and a strong correlation between $\kappa$ and $B$ can be identified.

Once prior and posterior distributions of input parameters are available, we can evaluate the corresponding distributions of the QoI $\tilde{U}(r)$ by performing uncertainty propagation. Samples are randomly generated based on prior and posterior distributions, and then propagated to the output $\tilde{U}(r)$ via surrogate model predictions. The corresponding output results are then gathered to obtain distributions of $\tilde{U}(r)$ (Figure 14).

The distribution of $\tilde{U}(r)$ obtained with prior prediction presents a significant level of uncertainty, and the one obtained with posterior prediction shows almost no uncertainty thanks to the reduction of uncertainties by data calibration. Even though uncertainties have been largely reduced on $\tilde{U}(r)$, local deviations compared to the reference data can still be observed. This indicates that the current configuration needs other improvements than data calibration to obtain better results.

We have seen that all possible combinations of $\kappa$ and $B$ allowed in posterior distributions give almost the same velocity profile, and the reason can be explained after a very simple manipulation of the law- 
of-the wall. Based on the log-law we can establish a relation between $\mathrm{K}$ and $B$ :

$$
B=-\frac{C_{1}}{\kappa}+C_{2}, C_{1}=\ln y^{+}, C_{2}=u^{+}
$$

From a LES with $\kappa=0.41$ and $B=5.5$ (located in the narrow zone of joint distribution in Figure 13), values of $C_{1}$ and $C_{2}$ can be determined such that $C_{1}=\ln (35)=3.55$ and $C_{2}=14.25$. In Figure 15 the curve of the equation (8) is plotted and it indeed passes through the middle of the joint distribution. Hence, a linear correlation exists between $B$ and $1 / \kappa$ which allows the error minimization on $\tilde{U}(r)$.
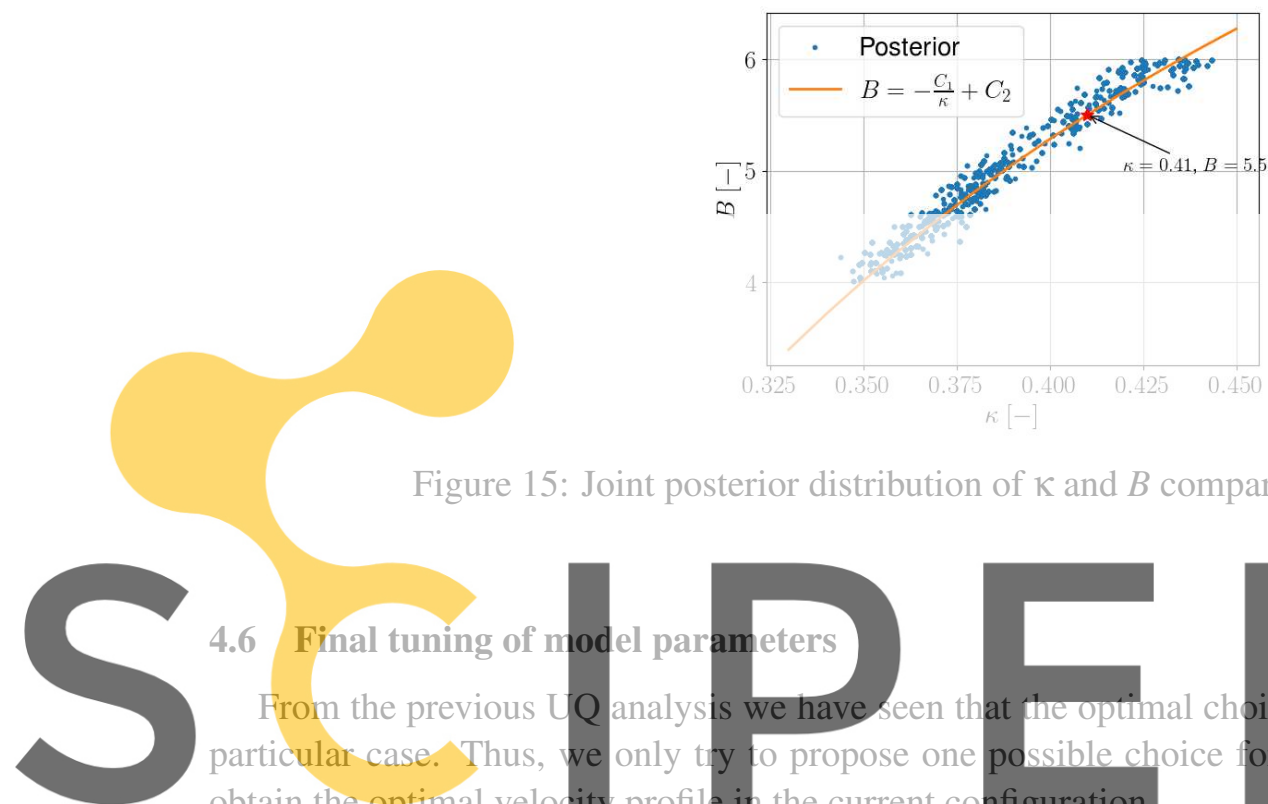

Figure 15: Joint posterior distribution of $\kappa$ and $B$ compared with equation (8)

4.6 Final tuning of nodel parameters

From the previous UQ

particular case. Thus,

obtain the optimal velocity profile in the current co
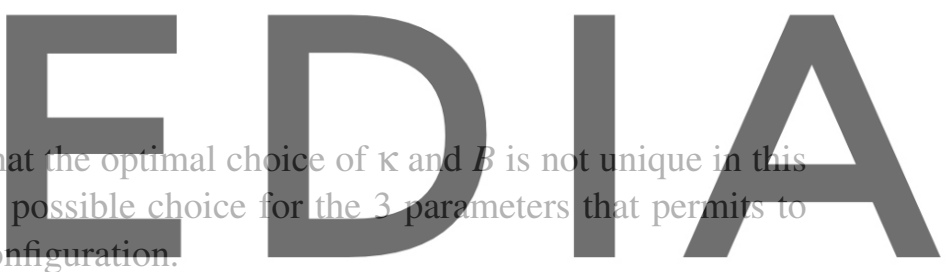

The sensitivity analysis in the Section 4.4 has shown that $K$ and $B$ are more critical parameters and

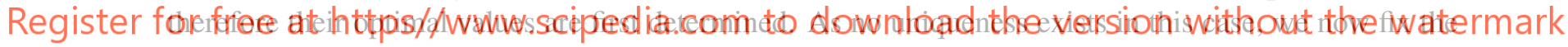
parameters $k=0.41$ and $B=5.5$ which are likely values as shown in Figure 15. To determine the optimal value of $C_{s}$ between 0.1 and 0.2 , we can either rely on LES to compute the error $\varepsilon_{\tilde{U}}$ for different values of $C_{s}$, or use the the surrogate model of $\varepsilon_{\tilde{U}}$ to predict the error for given $C_{s}$. Both approaches are adopted and the results are compared below.

Results of 11 LES are plotted in Figure 16. The variation due to the value of $C_{S}$ used is relatively small, as $C_{s}$ is the least influential parameter for QoIs. The corresponding error $\varepsilon_{\tilde{U}}$ from LES is presented in Figure 17 and compared with the predictions by the surrogate model of $\varepsilon_{\tilde{U}}$. Both of them show a similar evolution trend while deviations between them can reach at most $12 \%$. The minimum is located at 0.11 according to LES and at 0.12 based on the prediction of surrogate model. In this case we give more credit to LES results and the value chosen is 0.11 for $C_{s}$, though 0.12 would give almost the same result.

\section{CONCLUSIONS AND FUTURE WORK}

In this work, a UQ-CFD methodology was presented and applied to the LES of a turbulent pipe flow. The methodology consists of evaluating uncertainties from parameters of models used. UQ analysis is based on surrogate models of QoIs from LES, thus reducing the computational cost generated by 


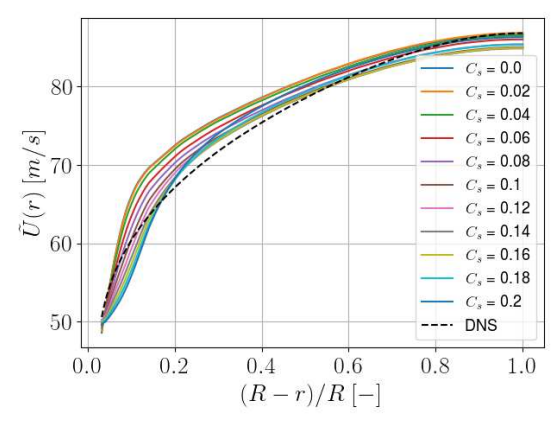

Figure 16: $\tilde{U}$ obtained using LES

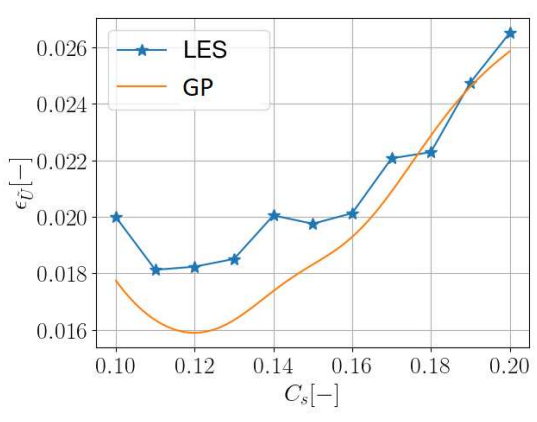

Figure 17: Comparison of $\varepsilon_{\tilde{U}}$ from LES and surrogate model

numerous LES in the application of UQ techniques. Different capabilities of the developed methodology are illustrated, including sensitivity analysis, data calibration and uncertainty propagation.

The analysis shows that parameters of the wall model are more critical in the pipe simulation, while the impact of the parameter of the SGS model increases near the wall. With the help of the data calibration, uncertainties on parameters are largely reduced as well as those on QoIs $\tilde{U}(r)$. A strong correlation is found between parameters of the wall-model, which is intrinsic to the model formulation. Following the UQ analysis, an optimal set of parameters is proposed which allows to minimize the errors on the velocity profile compared to the reference DNS data.

It is worth emphasizing that the proposed methodology, which combines Latin Hypercube sampling, surrogate modeling, sensitivity analysis and Bayesian data calibration is general and can be applied to any UQ problem involving complex computer codes. The application of such a methodology on a relatively simple case also allows us to anticipate difficulties when studying more complex cases.

In the future work, the application of current methodology on internal combustion engine simulation is envisaged, however some challenges stand out :

- In this study, a single LES of a pipe flow demands a runtime of 7 hours on 36 CPUs, in total around 250 CPU.hours. 400 experiments are used for building surrogate models, resulting in a total computational cost of $10^{5}$ CPU.hours. For more complex engine simulations, a single engine cycle requires much more computational resource of about $10^{4}$ CPU.hours, which makes the current methodology too expensive to use, even only for surrogate modeling.

- Unlike the pipe flow which is statistical stationary, in-cylinder engine flow is totally transient and presents variabilities between different cycles. Thus, QoIs extracted from a single cycle may not be representative of the global characteristics of the flow and values obtained from a certain number of cycles is preferred, which again increases the computational cost of a single experiment.

In our next studies we'd like to integrate an adaptive sampling strategy coupled with surrogate modeling with which DOE is not generated once but updated consecutively by adding critical samples to improve the quality of surrogate models efficiently. Also, qualitative studies will be carried out to investigate the main uncertainty sources in engine simulations and define quantifiable QoIs for UQ analysis.

\section{REFERENCES}

[1] $\mathrm{Wu}, \mathrm{X}$. and Moin, P. A direct numerical simulation study on the mean velocity characteristics in turbulent pipe flow. J. Fluid Mech., 608:81-112, 2008. 
[2] Bestion, D., et al. Review of uncertainty methods for CFD application to nuclear reactor thermalhydraulics. In NUTHOS 11- The 11th International Topical Meeting on Nuclear Reactor Thermal Hydraulics, Operation and Safety, Gyeongju, South Korea, October 2016.

[3] Schaefer, J. A., et al. Approaches for quantifying uncertainties in computational modeling for aerospace applications. In AIAA Scitech 2020 Forum, Reston, Virginia, 01062020, American Institute of Aeronautics and Astronautics.

[4] Tinoco, E. Validation and minimizing cfd uncertainty for commercial aircraft applications. In 26th AIAA Applied Aerodynamics Conference, Reston, Virigina, 08182008, American Institute of Aeronautics and Astronautics.

[5] Gainey, B., et al. A guide to uncertainty quantification for experimental engine research and heat release analysis. SAE Int. J. Engines, 12(5), 2019.

[6] Roustant, O., et al. Dicekriging, Diceoptim : Two R packages for the analysis of computer experiments by kriging-based metamodeling and optimization. J. Stat. Softw., 51(1), 2012.

[7] Garnier, E., et al. Large Eddy Simulation for Compressible Flows. Scientific Computation, Springer Netherlands and Springer e-books, Dordrecht, 2009.

[8] Pope, S. B. Turbulent flows. Cambridge University Press, Cambridge, 2000.

[9] Meyers, J. and Sagaut, P. On the model coefficients for the standard and the variational multi-scale smagorinsky model. J. Fluid Mech., 569:287-319, 2006.

[10] Zanoun, E.-S., et al. Evaluating the law of the wall in two-dimensional fully developed turbulent channel flows. Phys. Fluids, 15(10):3079, 2003.

[11] Eggels, J. G. M., et al. Fully developed turbulent pipe flow: a comparison between direct numerical simulation and experiment. J. Fluid Mech., 268:175-210, 1994.

[12] Baum, E., et al. On the validation of les applied to internal combustion engine flows: Part 1: Comprehensive experimental database. Flow, Turb. Combust., 92(1-2):269-297, 2014.

[13] Richards, K., et al. Converge (v2.4), convergent science. Madison, WI, 2017.

[14] McKay, M. D., et al. A comparison of three methods for selecting values of input variables in the analysis of output from a computer code. Technometrics, 42(1):55, 2000.

[15] Kleijnen, J. P. Kriging metamodeling in simulation: A review. Eur. J. Oper. Res., 192(3):707-716, 2009.

[16] Sobol/, I. Global sensitivity indices for nonlinear mathematical models and their monte carlo estimates. Math. Comput. Simul., 55(1-3):271-280, 2001.

[17] Tarantola, S., et al. Random balance designs for the estimation of first order global sensitivity indices. Reliab. Eng. \& Syst. Saf., 91(6):717-727, 2006.

[18] Kennedy, M. C. and O'Hagan, A. Bayesian calibration of computer models. J. Royal Stat. Soc. Ser. B Stat. Methodol., 63(3):425-464, 2001.

[19] Mastrippolito, F. Optimisation de forme numérique de problèmes multiphysiques et multiéchelles : application aux échangeurs de chaleur. Thesis, Université de Lyon, December 2018.

[20] Hastie, T., et al. The Elements of Statistical Learning, volume 1. Springer New York, New York, NY, 2001.

[21] Zagarola, M. V. and Smits, A. J. Mean-flow scaling of turbulent pipe flow. J. Fluid Mech., 373:3379, 1998.

[22] Prandtl, L. 7. bericht über untersuchungen zur ausgebildeten turbulenz. ZAMM - J. Appl. Math. Mech. / Zeitschrift für Angewandte Math. und Mech., 5(2):136-139, 1925. 